\title{
Use of cytoplasmic 5'nucleotidase for differentiating malignant from benign monoclonal gammopathies
}

\author{
G Majumdar, S E Heard, A K Singh
}

\begin{abstract}
Bone marrow smears from 15 patients with multiple myeloma, 15 patients with monoclonal gammopathy of undetermined significance (MGUS), and 15 control subjects were examined for the presence of cytoplasmic 5'nucleotidase (c5NT) in plasma cells. Plasma cell positivity for c5NT (mean and $95 \%$ confidence interval) in patients with multiple myeloma numbered $46.4 \%(38 \cdot 0-54 \cdot 8 \%)$, in those with MGUS it was $15 \cdot 3 \%(11 \cdot 1-$ $19.6 \%)$, and in control subjects it was $1 \cdot 2 \%(0 \cdot 3-2 \cdot 1 \%)$. These findings indicate that c5NT can be used to differentiate benign from malignant monoclonal gammopathies.
\end{abstract}

Cytoplasmic 5'nucleotidase (c5NT) is one of several phosphatases present in different types of cells. Mohandes and Hayhoe showed that a high percentage of plasma cells from patients with myeloma contain c5NT compared with a low number in normal controls. ${ }^{1} \mathrm{We}$ tested for the presence of plasma cell c5NT in patients with multiple myeloma, MGUS, and in control subjects to determine its usefulness in differentiating benign from malignant monoclonal gammopathies.

\section{Methods}

Bone marrow smears from 15 patients with multiple myeloma (male:female ratio $=9: 6$, mean age 68 years) were used for the study. Eight bone marrow samples had been taken at the time of diagnosis, three in the plateau phase, and four during relapse. Bone marrow plasma cell count in these patients was $5-77 \%$ and their serum paraprotein concentration was 9-80 g/l. Three patients with multiple myeloma had Bence Jones proteinuria. All 15 patients with MGUS (male:female ratio = $8: 7$, mean age 66 years) were under regular follow up for more than 20 months (range 21-45, median 28) and showed no significant increase in the paraprotein concentration over this period. Bone marrow plasma cell count in these patients was $1-7 \%$ and the serum paraprotein concentration was 3-26 g/l. Bone marrow smears from 15 patients (male: female ratio $=9: 6$, mean age 62 years) who were investigated for various complaints but showed no evidence of primary haematological disorder were used as controls. Bone marrow plasma cell count in control subjects was $1-3 \%$. All bone marrow samples were collected during the course of routine investigation when first seen or during follow up. Unfixed smears stored at room temperature for up to six months were used for the current investigation.

The presence of c5NT was shown by the method described by Hayhoe. ${ }^{2}$ Briefly, bone marrow smears fixed in acetone were incubated in barbitone buffer containing adenosine monophosphate (AMP), manganous acetate, tartaric acid and lead acetate for six hours at $37^{\circ} \mathrm{C}$. Smears were then fixed in formalin vapour and immersed in ammonium sulphide followed by counterstaining with methyl green. A second set of smears was similarly tested in parallel using $\beta$-glycerophosphate instead of AMP.

The stained smears were examined under a light microscope for the presence of dark brown granules in the cytoplasm. Cells showing only membrane bound granules were not counted. Plasma cells were categorised as negative or positive for c5NT. In all patients with multiple myeloma 100 plasma cells were examined, but in those with MGUS and in control samples, depending on the plasma cell count, 30-100 cells were examined in each smear. The results are expressed as percentage of plasma cells.

Statistical analysis was done by paired $t$ test.

\section{Results}

In patients with multiple myeloma $3-70 \%$ (mean $46.4^{\circ} \%, 95 \%$ confidence interval 38.0 $54.8^{\circ}{ }_{0}$ ) of plasma cells were c5NT positive. Only one patient had less than $33 \%$ positive cells. In the MGUS group the range of positive cells was $0-28 \%$ (mean $15.3 \%, 95 \%$ confidence interval $11 \cdot 1-19 \cdot 6 \%$ ) with only one patient having less than $6 \%$ of $\mathrm{c} 5 \mathrm{NT}$ positive cells. In control subjects the range was $0-5 \%$ (mean $1.2 \%, 95 \%$ confidence interval $0.3-$ $2 \cdot 1^{\circ}$ o). The differences between patients with multiple myeloma, those with MGUS, and controls were significant ( $p<0.001$ between each pair of groups). There was no correlation between the percentage of cells showing c5NT with total plasma cell count, paraprotein concentration, and in patients with multiple myeloma, with the stage of the disease.

Analysis of the results according to the scoring system proposed by Mohandes and Hayhoe ${ }^{1}$ showed a score of (mean and range) $125.5(26-179)$ in the group with multiple myeloma 28.1 (14-93) in the MGUS group, and $19 \cdot 7(0-50)$ in the controls. The differences 
between each pair of the results were significant ( $p<0.001)$. Only one patient with multiple myeloma had a score of less than 100 but six patients with MGUS had a score of less than 50 which was the highest score obtained in control subjects.

\section{Discussion}

Monoclonal gammopathy is common, especially in the elderly. Most of these patients fall in the MGUS group and are followed up indefinitely, though only a small percentage progress to malignant gammopathies. ${ }^{3}$ Several tests like the determination of serum $\beta$-2microglobulin and neopterin concentration, ${ }^{4}$ labelling index of plasma cells using either ${ }^{3} \mathrm{H}$ thymidine $^{5}$ or bromodeoxyuridine, ${ }^{6}$ scoring method using serum and bone marrow variables $^{7}$ and number of activated CD8 + cells $^{8}$ have been reported to be useful in the differentiation of malignant from benign gammopathies, but all have shown various degrees of overlap.

Other cytochemical methods like plasma cell acid phosphatase score $\mathrm{e}^{910}$ and leucocyte alkaline phosphatase score $^{10}$ also showed significant overlap between groups with multiple myeloma and groups with MGUS. All these tests and the scoring systems show a high degree of specificity but the sensitivity is often unacceptably low, especially in the plateau phase or during remission of multiple myeloma. At present long term follow up of the patients with MGUS is the only satisfactory method for determining the true prognosis. ${ }^{11}$

The study reported here shows the potential usefulness of determining c5NT in plasma cells with minimum overlap between multiple myeloma and MGUS groups. A discriminant level of $30 \%$ c $5 \mathrm{NT}$ positivity shows $93 \%$ sensitivity and $100 \%$ specificity for the diagnosis of malignant gammopathy. Patients with multiple myeloma even in plateau phase with low plasma cell counts and paraprotein concentrations showed a high degree of c5NT positivity. Conversely, patients with MGUS with moderately high paraprotein concentrations did not show a high degree of positivity.

The scoring system ( 0 for negative, 1 for membrane positivity, 2 for weak c5NT and 3 for strong $(5 \mathrm{NT})$, provided no advantage over the simple counting of c5NT positive cells. Though overlap between multiple myeloma and MGUS groups was similar to that obtained by $\mathrm{c} 5 \mathrm{NT} \%$, there was a greater degree of overlap between MGUS and control groups. As scoring involves subjective assessment of the degree of positivity, there is an obvious risk of observer bias. The scoring system includes membrane positivity which can be found in other cell types like T lymphocytes. Therefore, a simple count of c5NT positive cells seems to be a better method of assessment than the scoring system.

Determination of c5NT is a cheap and simple test which can be done on bone marrow aspirates taken for routine investigation of monoclonal gammopathy. As the smears remain usable for at least six months, even at room temperature, the test can be done in batches, thus reducing the overall cost of the test. It is therefore concluded that determination of c5NT of plasma cells is a useful test for differentiating malignant from benign monoclonal gammopathies.

1 El Mohandes EA, Hayhoe FGJ. 5'nucleotidase activity in myeloma cells. Clin Lab Haematol 1983;5:265-9.

2 Hayhoe FGJ. Cytochemistry of the acute leukaemias. Histochem J 1984;16:1051-9.

3 Malacrida V, De Francesco D, Banfi G, Porta FA, Riches PG. Laboratory investigation of monoclonal gammopathy during 10 years of screening in a general hospital. $J$ Clin Pathol 1987;40:793-7.

4 Fine JM, Lambin P, Desjobert H. Serum neopterin and beta-2-microglobulin concentration in monoclonal gammopathies. Acta Med Scand 1988;224:179-82.

5 Boccadoro M, Gavarotti P, Fossati G, Pileri A, Marmont F, Neretto G. Low plasma cell 3(H)-thymidine incorporation in monoclonal gammopathy of undetermined signifin (MGS), smouldering myeloma and remission nificance (MGUS), smouldering myeloma and remission phase myeloma: a reliable indicator of patien.

6 ing therapy. Br J Haematol 1984;58:689-96. fluorescence labelling indices in myeloma and related fluorescence labelling indices in myeloma and related monoclonal

7 Morell A, Maurer W, Skvaril F, Barandun S. Differentiation between benign and malignant monoclonal gammopathies by discriminant analysis on serum and bone marrow parameters. Acta Haematol 1978;60:129-36.

8 Dianzani U, Pileri A, Boccadoro M, et al. Activated idiotype-reactive cells in suppressive/cytotoxic subpopulation of monoclonal gammopathies: correlation with diagnosis and disease status. Blood 1988;72:1064-8.

9 Hoffmann JJML, Breed WPM, Swaak-Lammers N. Plasma cell acid phosphatase score in multiple myeloma and related disorders. Br J Haematol 1985;59:67-72.

10 Quaglino D, Hayhoe FGJ. Haematological cytochemistry. 2nd edition. Edinburgh: Churchill Livingstone, 1988: 2nd ed.

11 Kyle RA. Benign monoclonal gammopathy: a misnomer? JAMA 1984;251:1849-54. 